\title{
A 3D printed cast for minimally invasive transfer of distal radius osteotomy: a cadaver study
}

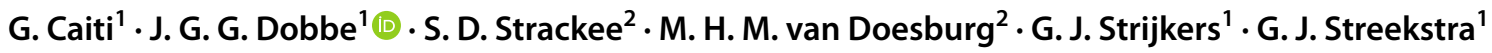

Received: 26 October 2020 / Accepted: 3 January 2021 / Published online: 21 January 2021

(c) The Author(s) 2021

\begin{abstract}
Purpose In corrective osteotomy of the distal radius, patient-specific 3D printed surgical guides or optical navigation systems are often used to navigate the surgical saw. The purpose of this cadaver study is to present and evaluate a novel cast-based guiding system to transfer the virtually planned corrective osteotomy of the distal radius.

Methods We developed a cast-based guiding system composed of a cast featuring two drilling slots as well as an external cutting guide that was used to orient the surgical saw for osteotomy in the preoperatively planned position. The device was tested on five cadaver specimens with different body fat percentages. A repositioning experiment was performed to assess the precision of replacing an arm in the cast. Accuracy and precision of drilling and cutting using the proposed cast-based guiding system were evaluated using the same five cadaver arms. CT imaging was used to quantify the positioning errors in 3D. Results For normal-weight cadavers, the resulting total translation and rotation repositioning errors were $\pm 2 \mathrm{~mm}$ and $\pm 2^{\circ}$. Across the five performed surgeries, the median accuracy and Inter Quartile Ranges (IQR) of pre-operatively planned drilling trajectories were $4.3^{\circ}\left(\mathrm{IQR}=2.4^{\circ}\right)$ and $3.1 \mathrm{~mm}(\mathrm{IQR}=4.9 \mathrm{~mm})$. Median rotational and translational errors in transferring the pre-operatively planned osteotomy plane were and $3.9^{\circ}\left(\mathrm{IQR}=4.5^{\circ}\right)$ and $2.6 \mathrm{~mm}(\mathrm{IQR}=4.2 \mathrm{~mm})$, respectively.

Conclusion For normal weight arm specimens, navigation of corrective osteotomy via a cast-based guide resulted in transfer errors comparable to those using invasive surgical guides. The promising positioning capabilities justify further investigating whether the method could ultimately be used in a clinical setting, which could especially be of interest when used with less invasive osteosynthesis material.
\end{abstract}

Keywords Radius osteotomy $\cdot$ Surgical guide $\cdot 3 \mathrm{D}$ printing $\cdot$ Minimally invasive navigation $\cdot$ Computer-assisted orthopedic surgery

\section{Introduction}

Distal radius malunion is a complication frequently arising after a conservatively treated fracture of the distal radius [1]. When clinically relevant, the malunion is surgically treated by corrective osteotomy $(\mathrm{CO})$ surgery [2]. The purpose of $\mathrm{CO}$ is to reconstruct as best as possible the original bone alignment by cutting the bone at the level of the old fracture

J. G. G. Dobbe

j.g.dobbe@amsterdamumc.nl

1 Department of Biomedical Engineering and Physics, Amsterdam Movement Sciences, Amsterdam UMC, University of Amsterdam, Amsterdam, The Netherlands

2 Department of Plastic, Reconstructive and Hand Surgery, Amsterdam Movement Sciences, Amsterdam UMC, University of Amsterdam, Amsterdam, The Netherlands (osteotomy) and then repositioning and fixating the bone segments to a near-anatomical alignment. State-of-the-art $\mathrm{CO}$ techniques combine the utilization of three-dimensional (3D) pre-operative virtual planning and 3D printed patientspecific instruments (PSI's). With virtual pre-operative planning, 3D virtual models of the affected and the contralateral healthy radius anatomy are generated from a bilateral computed tomography (CT) scan of a patient's forearm [3]. Furthermore, starting from these 3D virtual models, it is possible to design PSI's exactly fitting the bone anatomy and featuring a cutting slit and drilling holes to orient the surgical tools as preoperatively planned [4-7]. Currently, the use of PSI's is growing in popularity because they offer a straightforward method for surgical navigation [8], they are accurate in transferring the virtual plan to the patient [9], and are becoming more affordable thanks to advances in 
design software and developments in additive manufacturing technology [10].

Recently, a minimally invasive patient-specific rimmed wedge implant has been proposed for repositioning and fixation in corrective osteotomy of the distal radius, which exactly fits the osteotomized bone segments in the planned position [11]. Although far from being used in a clinical setting, this procedure requires accurate osteotomy, since the wedge fits in between the bone segments at a single planned location. The osteotomy in this approach could be performed using PSI's. However, a drawback of PSI's is that complete dissection of the soft tissues covering the bone surface is generally required in order to ensure an accurate fit of the PSI on the target bone. This is undesired because with complete soft tissue dissection the patient is expected to experience slower recovery, inferior functional results and a poorer forearm appearance after surgery [12,13] (Level of evidence IV). A navigation technique that positions and orients the surgical saw without considerable soft tissue dissection would better fit the minimally invasive approach of corrective osteotomy using a rimmed wedge implant.

One of the possible approaches to minimize the invasiveness of the osteotomy procedure and to eliminate the need for a large incision is to use an external cutting guide to be mounted on percutaneously inserted pins. Percutaneous pinning is a common procedure in hand surgery and is frequently performed in order to fixate carpal or distal radius fractures [14]. Systems for minimal invasive navigation of percutaneous pinning of carpal bones have been previously proposed [15-17]. In systems targeting percutaneous pinning of the scaphoid, the wrist is set in a fixed position through a stabilization platform or stereotactic frame which features a local coordinate reference (LCR). Then, image-guided surgical navigation can be performed by tracking the LCR on the wrist stabilization frame during image-guided surgery [15-17]. These systems permit minimally invasive navigation because markers are not directly attached to the bone but to the wrist-stabilization frame. However, the positioning accuracy of these stabilization platforms is often unknown $[16,17]$ or suffers from large patient-device motion caused by drilling (up to on average $16 \mathrm{~mm}$ and $6.5^{\circ}$ ) [15].

The aim of this cadaver study is to propose and evaluate a minimally invasive method for percutaneous pinning that serves to place a cutting guide for navigation of the surgical saw for corrective surgery of the distal radius. The proposed method consists of a 3D-printed cast that enables percutaneous pin insertion and positioning of an external cutting guide that is slid over the pins after cast removal. We evaluate the repeatability of repositioning the radius in the cast and its dependency on the amount of soft tissue surrounding the bone. We further evaluate the accuracy and precision of transferring the virtual pins and the osteotomy cut using the proposed surgical system.

\section{Materials and methods}

The proposed method is based on the following workflow (Fig. 1): After positioning the arm in a reproducible position (detailed in section 2a), a preoperative bilateral CT scan is acquired. Then, the $3 \mathrm{D}$ pre-operative planning is performed as described in [18-22], where the osteotomy plane and bone repositioning are defined. Next, the cast and the external cutting guide are designed, via Computer Aided Design

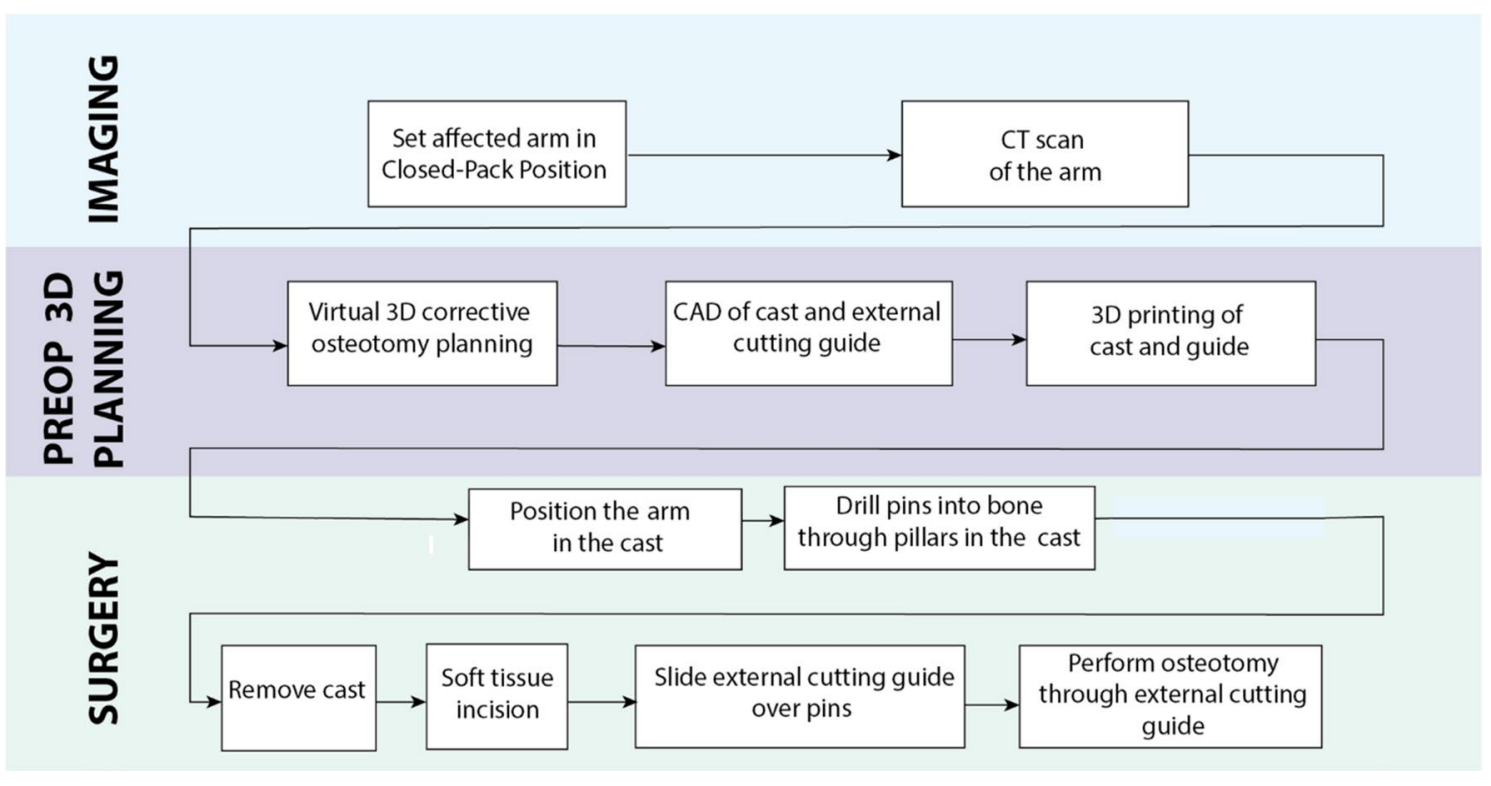

Fig. 1 Pre and intra-operative workflow for cast-guided osteotomy 
(CAD) software, and 3D printed. In this study, image analysis and 3D printing took approximately $24 \mathrm{~h}$. During surgery, the cast is applied. Percutaneous pinning of the radius is performed through drilling pillars integrated on the cast surface. After cast removal and preliminary incision of the skin and soft tissues, the external cutting guide is placed over the pins and the osteotomy can be performed through a slit in the guide.

Figure 2 shows steps in the surgical procedure.

In the following subsections, the rationale behind the cast design (A), the experiments to investigate the reproducibility of positioning the arm in the cast (B), and the method to quantify the surgical transfer accuracy $(\mathrm{C})$ are presented.

\section{Cast and guide design}

In this study, five cadaver arm specimens were included. Each cast-based surgical guide was designed starting from a CT image of a fresh-frozen cadaver arm after thawing for $24 \mathrm{~h}$. One of the main challenges of the presented method was to reproduce the preoperatively scanned position of the radius relative to the cast during surgery. Therefore, a stable and reproducible forearm position was chosen when imaging the arms. A high reproducibility of positioning was expected in the so-called Closed-Pack Position (CPP) in which the contact surface between articulating bones is maximal resulting in the highest mechanical stability. For the wrist joint a CPP is obtained with the wrist in maximal (approx. $35^{\circ}$ ) ulnar deviation. In order to avoid shifting of the cast along the radial axis, we further chose to flex the fingers and the elbow at approx. $45^{\circ}$ (Fig. 3a). After

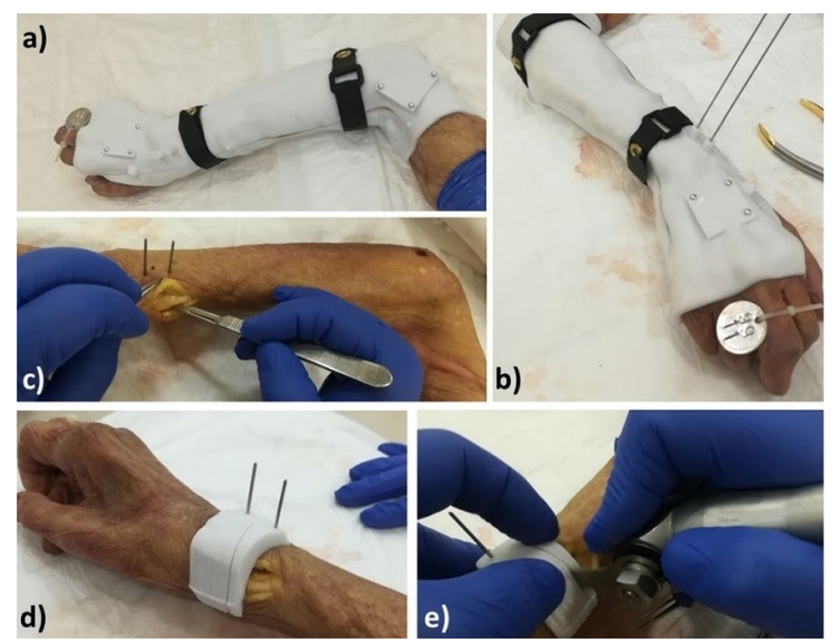

Fig. 2 Practical steps during the surgical procedure. a The arm is inserted in the cast; $\mathbf{b}$ two self-drilling/self-tapping pins are percutaneously drilled through the drilling slots; $\mathbf{c}$ after cast removal, a small incision is performed; $\mathbf{d}$ The external cutting guide is slid over the previously fixed pins; e osteotomy is performed thorough the slit in the cutting guide
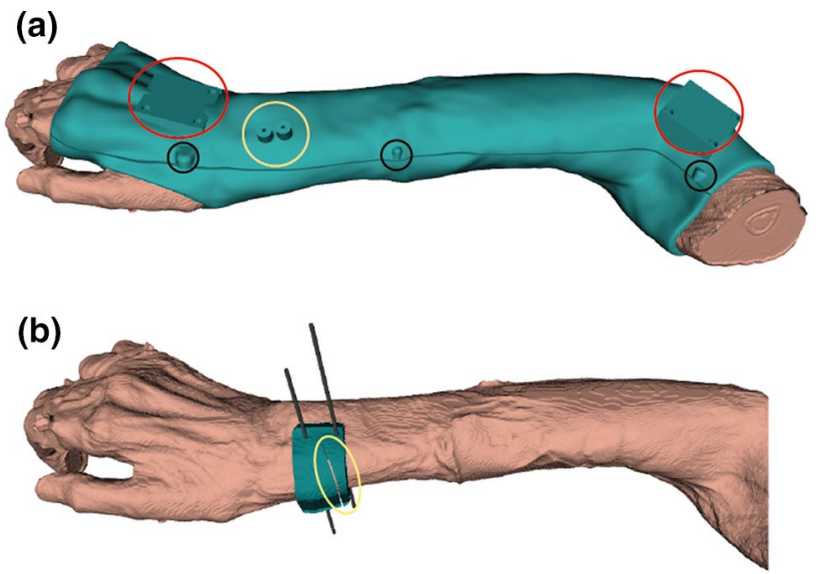

Fig. 3 a The arm is placed in a stable and reproducible position (ulnar deviation $\sim 35^{\circ}$, fingers flexed and elbow at $\sim 45^{\circ}$ flexion). The cast features distal and proximal marker tools (red circle); two drilling pillars (yellow circle) and snap-lock pins (black circles); b after inserting self-drilling/self-tapping pins into the bone through the pillars in the cast, and after cast removal, an external cutting guide is slid over the pins. The guide features a slit (marked in yellow) to guide the surgical cutting blade

arm replacement $(10 \times)$, a scan was acquired with either a Brilliance 64-channel scanner (Philips Healthcare, Best, The Netherlands) or with a Sensation 64-channel scanner (Siemens, Munich, Germany). Comparable dedicated scanning protocols were adopted for the two scanners (tube voltage $120 \mathrm{kVp}$; exposure: $500 \mathrm{mAs}$; slice thickness $0.90 \mathrm{~mm}$ ) and (Tube Voltage $120 \mathrm{kVp}$; exposure: $400 \mathrm{mAs}$; slice thickness $0.75 \mathrm{~mm}$ ) with isotropic voxel spacing of $0.45 \mathrm{~mm}$, respectively. From each acquired CT scan, the radius bone and the entire arm were segmented using custom software, as described in [23]. Each cast was designed via Autodesk (Autodesk Inc., San Rafael, California, USA) and was composed of two matching halves. In order to fit the two halves together intraoperatively, 6 snap-lock aluminum pins ( 3 on the ulnar and 3 on the radial side of the cast) were placed on the cast edge (Fig. 3a). Two marker tools were included in each cast, representing a distal and proximal Local Coordinate System (LCR). Each marker tool contained three aluminum spheres ( $5 \mathrm{~mm}$ diameter) in an orthogonal configuration. These local coordinate systems served to quantify deformation of the cast and positioning of the radius bone with respect to the cast when repetitively placing the arm in the cast (detailed in section B). Each cast featured two pillars to guide percutaneous pin insertion targeting the dorsal aspect of the distal radius. To this end, self-drilling/selftapping pins (Stryker, Michigan, United States, diameter $3 \mathrm{~mm}$, total length $80 \mathrm{~mm}$, thread length $20 \mathrm{~mm}$ ) were used. Drilling pillars were placed parallel to each other and perpendicular to the normal of the chosen cutting 
plane (Fig. 3a). This made the osteotomy cut irrespective of guide placement along the pins. Care was taken to make cast removal feasible for each of the applicable drill paths. For osteotomy guidance, external cutting guides were designed (Fig. 3b) using the same custom software as described above. Both casts and external guides were 3D printed in Polycarbonate-ISO (PC-ISO, certified ISO 10,993, United State Pharmacopeia -USP-Class VI), with a Stratasys Fortus 450 mc Fused Deposition Modelling (FDM) printer (Stratasys, Eden Prairie, Minnesota, USA). Printer accuracy was $\pm 0.127 \mathrm{~mm}$ in all directions. The thickness of the cast was set to $4 \mathrm{~mm}$. The material PC-ISO is sterilizable using gamma radiation or ethylene oxide gas [24].

\section{Reproducibility of arm replacement}

Each cadaver specimen was placed and removed 10 times in the 3D printed cast to quantify the reproducibility of radius positioning relative to the cast. After each arm placement, a CT scan was acquired. The 3D polygonal model of the radius bone (see section A) was registered to each CT scan [23]. In agreement with previously reported study [9], a radius right-handed coordinate system (RCS) was defined for each segmented radius (see section A). In brief, the $z$-axis coincided with the principal axis of inertia of the radius model, the $\mathrm{x}$-axis was directed toward the styloid process and the y-axis was consequently oriented to establish an orthogonal triplet of vectors (Fig. 4). Markers tools on the surface of the cast were used to provide local CS's for the cast. In brief, the metal spheres included in the marker tools were segmented by automatic thresholding which enabled automatic extraction of the orthogonal axes that define the local coordinate systems for the two marker tools, since the distance between the three spheres in each marker tool was unique, as described in [19]. In this way a proximal coordinate system (PCS) and a distal coordinate system (DCS) were defined on the cast (Fig. 4). The DCS served as reference coordinate system for expressing:

1. The relative displacement of the radius into the cast, represented by variability in the RCS-to-DCS transformation

2. The deformation of the cast during the experiment, represented by changes in the PCS-to-DCS transformation.

Each transformation matrix enabled calculations of the repositioning parameters, in six degrees of freedom (DOF) (3 rotations and 3 translations). Repetitive arm placements provided the reproducibility in the repositioning parameters and cast deformation. Errors are reported as the difference from the mean parameter.

The total translation $(\Delta T)$ and the total rotation $(\Delta R)$ errors, respectively, defined as the Euclidian norm of the translation and rotation parameters [25], were also calculated. We hypothesized that positioning of the radius in the cast and the subsequent drilling and cutting accuracy are dependent on the amount of rigid bony protrusions providing stability to the cast. The reproducibility may therefore deteriorate if more soft tissue covers these bony protrusions. In order to confirm our hypothesis, we report the positioning errors as a function of the soft-tissue percentage $\left(S T_{\%}\right)$ of the arm specimen, which was calculated as follows:

$S T_{\%}=\frac{V_{\text {arm }}-V_{\text {bone }}}{V_{\text {arm }}} \cdot 100 \%$

where $V_{\text {arm }}$ is the total volume of the segmented arm and $V_{\text {bone }}$ is the volume of all the segmented skeletal structures in the specimen. Mid-upper arm circumference was also used as an estimate of the body mass index (BMI) of the subjects [26]. Selection of the arm specimens was therefore based on the soft tissue content of the arms to obtain a range of softtissue percentages among the set of specimens.
Fig. 4 For each CT image, after segmentation, three Coordinate Systems (CS's) are defined: an anatomical CS for the radius bone (RCS) and CS's for the distal and for the proximal marker tools (DCS and PCS) on the cast. The transformation from RCS-to-DCS provides the relative position of the radius with respect to the cast. The transformation from PCSto-DCS represents the cast deformation

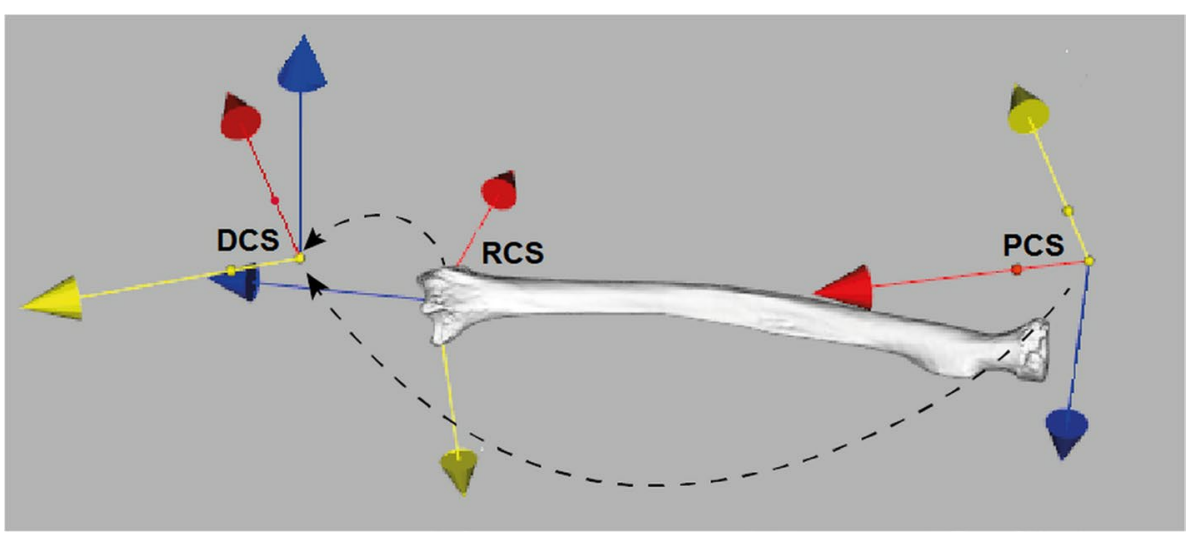




\section{Quantifying cutting and drilling error}

For each of the specimens, the overall procedure consisting of drilling through the cast and then performing the osteotomy through the external cutting guide was carried out by an experienced surgeon. After removal of the pins, a final CT scan of the drilled and cut bones was acquired. The images were analyzed in the following way: the distal and proximal bone segments were segmented and registered to the preoperative image that was used for planning. This enabled comparisons of the achieved drill-hole positions with the planned positions, as well as the achieved osteotomy cut with the planned cut. To this end, a distal and a proximal line was manually fit through the respective screw entry and exit points on the bone in 3D space. The difference between the planned and achieved pin placement was calculated as the distance $\left(\mathrm{dL}_{\mathrm{err}}\right)$ between the planned and achieved entry point of the drilling lines into the bone and as the angular offset ( $\left.\alpha_{\text {err }}\right)$ between the line directions (Fig. 5a). To quantify the osteotomy error, the achieved osteotomy plane was identified by manually placing three points on the surface of the proximal bone segment and then fitting a plane through these points. The difference between the planned and actual cutting plane was calculated as the distance $\left(\mathrm{dP}_{\text {err }}\right)$ between the centroids of the planned and the achieved bone cross sections [27] and as the angular error $\left(\beta_{\text {err }}\right)$ between the normals of the planned and the achieved cutting plane (Fig. 5b).

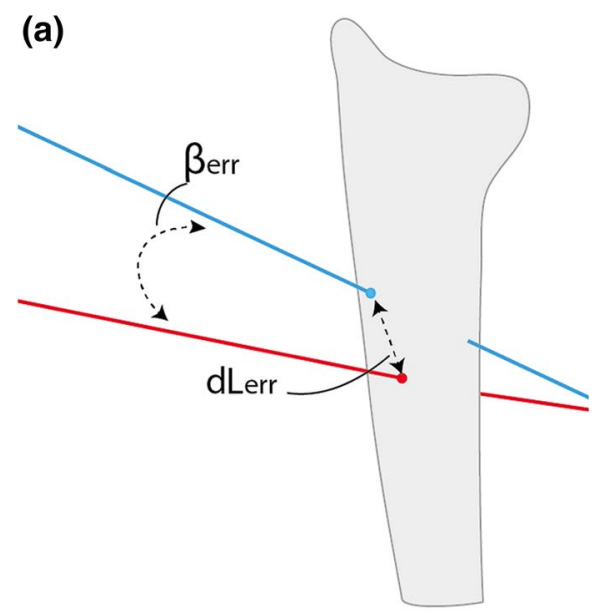

Fig. 5 a The planned (blue) and the achieved (red) drilling lines. The displacement error $\left(\mathrm{dL}_{\text {err }}\right)$ is defined as the distance between the entry point of each line into the bone polygon, the angular error $\left(\alpha_{\mathrm{err}}\right)$ is defined as the angle between the two line versors; $b$ the planned (blue) and the achieved (red) cutting planes and the corresponding

\section{Results}

\section{Reproducibility of arm positioning}

Quantification of the arm and bone volume from the CT scans of the five cadaver specimens, provided a soft tissue (ST) content of, respectively, $78.7 \%, 79.1 \%, 81.3 \%, 85.6 \%$ and $91.4 \%$. The second and the third arms (in order of ST content) originated from the same individual. Mid-upper arm circumference values were correlated with BMIs of 14 , $23,23,28$ and 31 which roughly categorize the subjects as, respectively, falling in the underweight, normal $(2 \times)$, overweight and obese ranges, according to [28]. For equivalent judgment of the 6 DoF parameters all left radii were mirrored. Boxplots reporting the median and the Interquartile Range (IQR) of the three translation parameters $\left(\Delta_{x}, \Delta_{y}, \Delta_{z}\right)$ and of the three rotation parameters $\left(\Delta_{\varphi_{x}}, \Delta_{\varphi_{y}}, \Delta_{\varphi_{z}}\right)$ are reported in Fig. 6a, b, respectively. The total rotation and translation errors obtained with the casts in the five cases are shown in Fig. 6c, d. While positioning errors appear to remain in the range $\pm 2 \mathrm{~mm} / \mathrm{deg}$ for specimens composed up to $81.3 \%$ by ST, we can see an increase in the positioning errors for the specimens categorized as overweight and obese ( 85.6 and $91.4 \%$ ST).

Variability of arm repositioning was partially caused by elastic deformation of the cast devices. Figure 7 shows summary statistics of the total translations and total rotations errors occurring between the marker tool coordinate systems (DCS and PCS) on the five casts during the repositioning experiment. Deformation of the cast contributed up to $2 \mathrm{~mm}$ to the overall repositioning translation error and

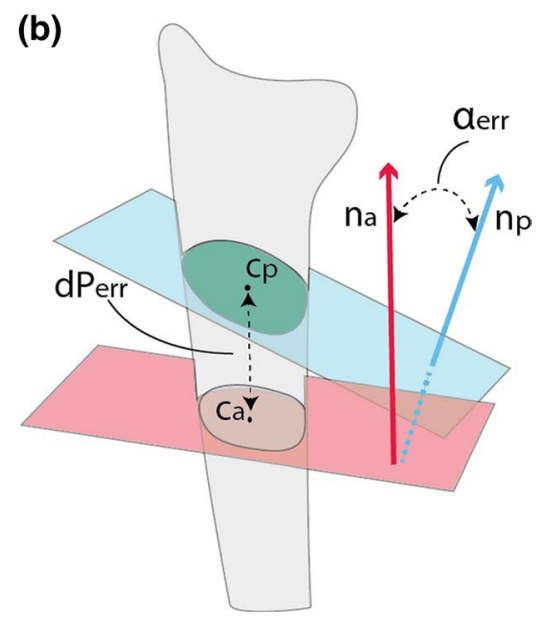

normal vectors $\left(n_{\mathrm{a}}, n_{\mathrm{p}}\right)$. The angular error $\left(\beta_{\text {err }}\right)$ is defined as the angle between $n_{\mathrm{a}}$ and $n_{\mathrm{p}}$. The distance between the centroids $\left(c_{\mathrm{a}}, c_{\mathrm{p}}\right)$ of the achieved and the planned bone cross sections represents the displacement error $\left(\mathrm{dP}_{\mathrm{err}}\right)$ 


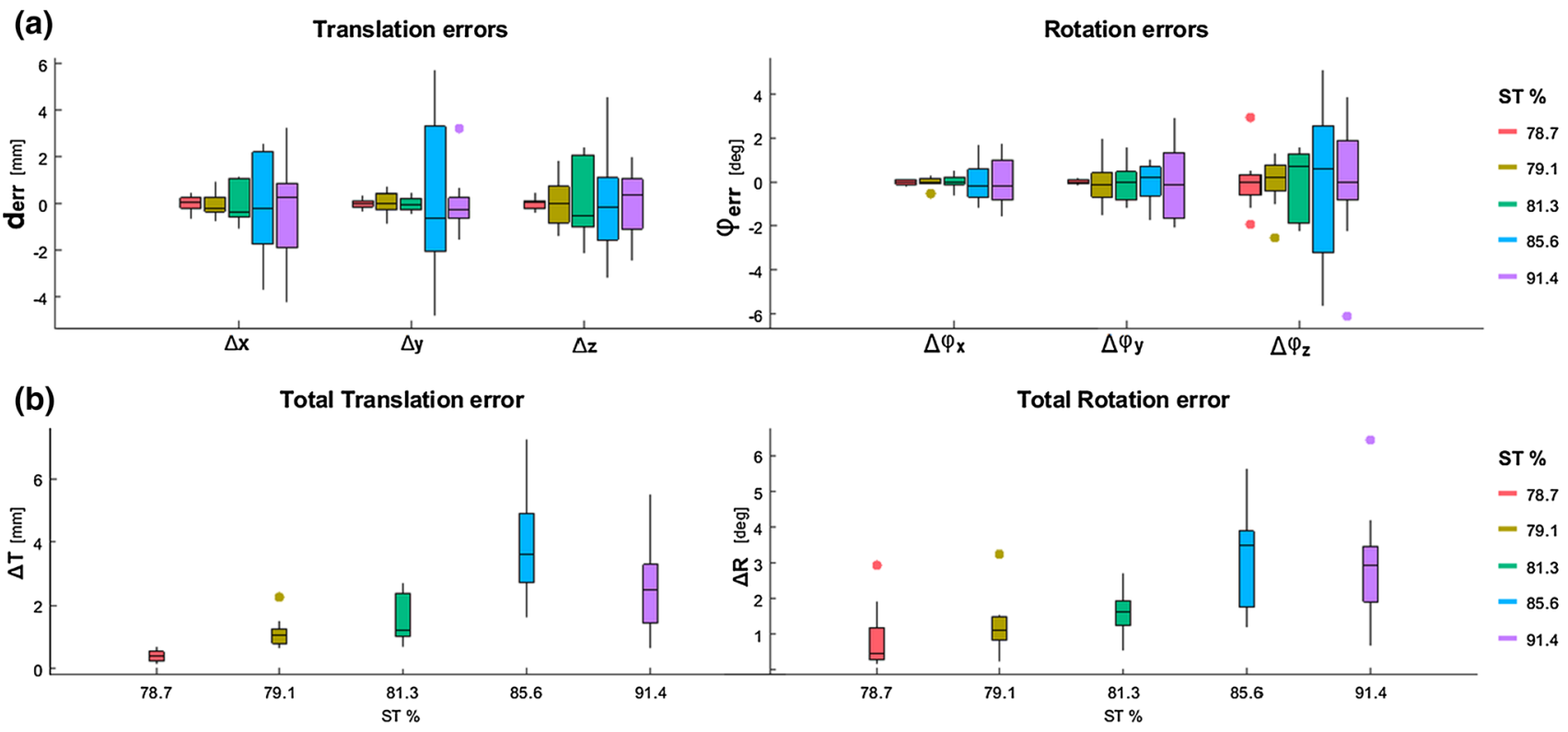

Fig. 6 Boxplots representing the summary statistics (median and IQR) of the 6 DoF repositioning errors in the 5 patient-specific casts, using specimens with increasing percentage of soft tissue (ST). On each box, the central mark indicates the median; the whisk- ers represent the 25th $(\mathrm{Q} 1)$ and 75 th $(\mathrm{Q} 3)$ percentiles range of each parameter. The dots are data considered outliers $\left(<\mathrm{Q} 1-1.5^{*} \mathrm{IQR}\right.$ or $\left.>\mathrm{Q} 3+1.5^{*} \mathrm{IQR}\right)$.: a The $6 \mathrm{DOF}$ translational rotational errors; $\mathbf{b}$ The total translation and rotation errors
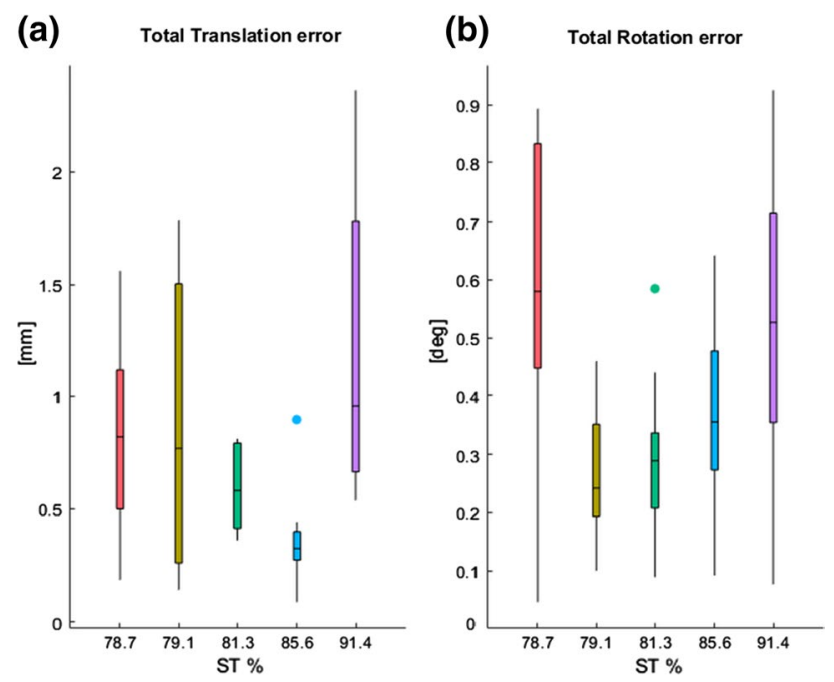

Fig. 7 Cast deformation represented by box-plot showing a) the total translation error $(\Delta T)$ and total rotation error $(\Delta R)$ occurring between the two local marker tools on the cast

up 1 degree to the total rotation error. No trend could be observed between deformation errors and specimens' soft tissue content (ST\%).

\section{Transfer error of drilling and cutting}

The five cast-guided osteotomies were performed as described in section 2a. In one case (91.4\% ST specimen),
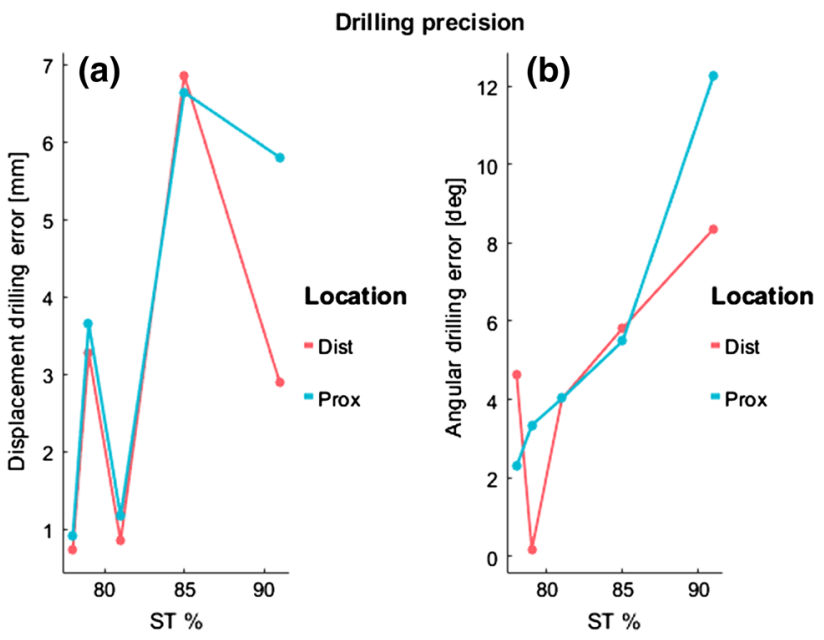

Fig. 8 Error parameters in transferring the drill holes to the specimens as a function of soft tissue percentage (ST\%). a Displacement error of the achieved drilling lines (proximal and distal) from the planned position; b Angular error between the planned and the achieved drilling lines

the drilling pillars were too tight for the chosen pins. Therefore, in the attempt to enlarge them, the pillars were damaged by the surgical drill. No plastic debris of the guide was observed at the surgical site in any of the cases. The errors in achieving the planned drilling trajectories across the five cases are depicted in Fig. 8. Displacement errors were similar for distal and proximal drilling lines in the same subject. 
An exception can be seen in the case of the specimen with 91.5\% ST, which could be explained by the damage caused to the drilling pillars of the cast. For cases up to $81.3 \%$ ST, displacement of drilling lines from the planned position did not exceed $4 \mathrm{~mm}$. Angular drilling errors were below $6^{\circ}$ for all the cases where the drilling guide remained intact.

The error in transferring the osteotomy through the custom cutting block in the selected cases is described in Fig. 9. Displacements errors in performing the planned osteotomy (Fig. 9a) were up to $4.5 \mathrm{~mm}$ for cases up to $81.3 \%$ ST. Angular errors (Fig. 9b) remained below $4.2^{\circ}$ in all the cases except for the $91.5 \%$ ST.

\section{Discussion}

The aim of the present cadaver study was to propose and evaluate a novel minimally invasive approach to quickly and easily navigate a corrective osteotomy of the distal radius. We designed an innovative 2-step surgical system composed of an externally fitting cast to guide dorsal insertion of two percutaneous self-drilling/self-tapping pins in the distal radius, and an external cutting guide to be slid over the pins. We presented the device together with the step-by-step surgical procedure which was performed on five cadaver specimens.

The error of repositioning the radius within the cast was evaluated in 6 Degrees of Freedom with a CT-based technique. In specimens with a normal body weight, the repositioning error was in the range $\pm 2 \mathrm{~mm}$ and $2^{\circ}$. This is comparable to the errors obtained when manually positioning invasive guides on the volar radial surface as quantified in
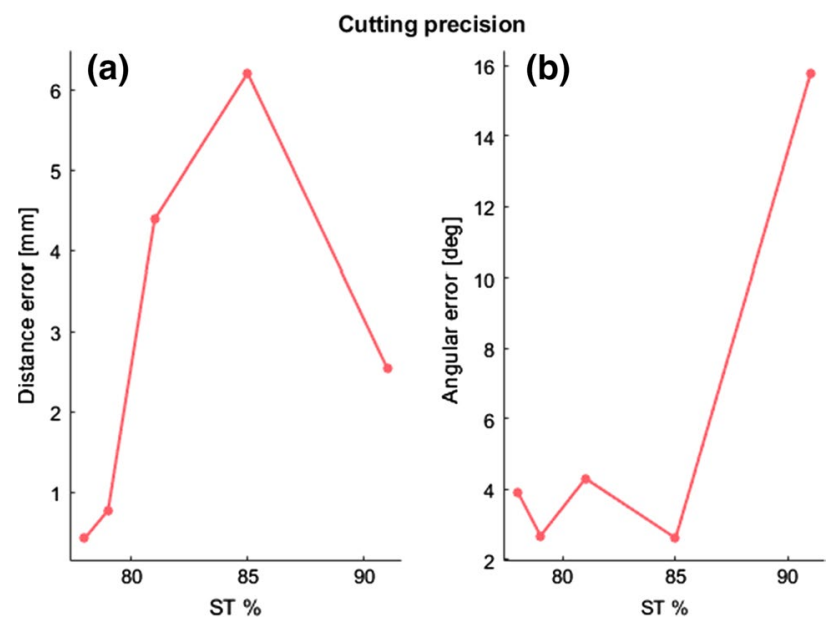

Fig. 9 Error in transferring the osteotomy across the five specimens with increasing percentage of soft tissue (ST), a distance error between the centroids of the bone cross-sections achieved with the planned and the achieved cutting planes; $\mathbf{b}$ angular error between the normal vector of the planned and the achieved osteotomy planes our previous study with the same image-based technique [9]. Except for one case (95.1 ST \%) where a surgical error damaged the drilling guide on the cast, the technique performed particularly well in achieving the planned osteotomy with errors limited to $4.4 \mathrm{~mm}$ and $4.2^{\circ}$. Errors in achieving the correct osteotomy orientation were smaller than the angular drilling errors. This reduced error could be explained by the use of the cutting block, which probably corrected the pins parallelism while performing the bone cut. In normal weight subjects, the proposed device has therefore the potential to render corrective osteotomy surgery less invasive, especially if used in combination with minimally invasive osteosynthesis material such as a custom wedge implant [11]. This patient-specific titanium wedge insert features two rims to stabilize the bone segments, and two drilling holes for screw fixation. The implant is designed to align the bone segments as preoperatively planned. Further study is needed to demonstrate the overall feasibility and accuracy of such an approach.

In this cadaver study, we mainly focused on the error of performing an osteotomy using a minimally invasive 3D printed cast. The positioning results seem promising, which justifies further investigating navigated osteotomy surgery using the cast-based guiding system in future research. However, the method is not ready for clinical use. There are several limitations that require further investigation. In our study, we relate positioning reliability to soft tissue volume while skin motion may have an independent influence that is worthwhile investigating. Although the proposed method for bone repositioning seems promising, it performed clearly better in specimens with a lower fat content. Deformation of the cast itself (up to $2 \mathrm{~mm}, 1^{\circ}$ ) contributed to the total error that we observed. Investigating alternative cast materials with better mechanical properties and using different designs of the cutting block may contribute to improved accuracy of the method. In this study we used a limited number of cadaver specimens. This hampers drawing a final conclusion on the clinical value of the method. For future research we therefore recommend optimizing cast design, avoiding obstruction of the surgical target giving a better surgical approach that avoids tendon or nerve injury, and evaluation of these techniques using more cadaver specimens. We also recommend investigating advantages, disadvantages, and potential risks and complications related to using the proposed cast-based navigation technique, and to compare these with established navigations techniques, including invasive guides and optical navigation. If the method is ultimately suitable for clinical evaluation, we recommend investigating whether the minimally invasive approach is substantially better from the patient's perspective compared to using invasive techniques.

The main challenge of this study was related to the error of repositioning the radius in the custom cast, which was 
designed to fit a deformable arm. Similar attempts of fitting external surgical guiding systems to the patient body have been reported in the literature. However, despite a favorable clinical outcome, the positioning accuracy of the devices has rarely been reported. For example, Li et al. used 3D printed PSI's to guide percutaneous vertebroplasty with a customized template that was fitting the back of the patient and featured drilling sleeves to guide the needle tip [29]. The customized template was used in combination with C-arm fluoroscopy in one patient case with good surgical outcome, but no methodological drilling errors were reported. Yin et al. reported the use of a 3D printed short cast extending to the wrist of the patient to guide percutaneous pinning for scaphoid fracture fixation [30]. Although design details and the surgical procedure were extensively described, neither postoperative evaluation nor the fixation error were quantified. Our study is therefore the first quantitative evaluation of the feasibility of using an external guiding device. Furthermore, compared to the previously reported studies our method does not expose subjects to intraoperative radiation.

\section{Conclusions}

We developed a minimally invasive device for the transfer of a 3D planned corrective osteotomy of the distal radius and evaluated the method experimentally. For cadaver specimens with a low and normal soft tissue content, precision in positioning the arm in the cast was comparable with the positioning accuracy of invasive PSI's for the distal radius. The promising positioning capabilities justify further investigating whether the method could ultimately be used in a clinical setting.

Acknowledgements The authors would like to thank P. Boomsma and the Department of Medical Innovation and Development for the help in the design and creation of the casts and guides, and Dr. A. Peymani from the Department of Plastic, Reconstructive and Hand Surgery for his active participation in the experiments.

\section{Compliance with ethical standards}

Conflict of interest The authors declare that they have no conflict of interest.

Ethical approval All procedures performed in studies involving human participants were in accordance with the ethical standards of the institutional and/or national research committee and with the $1964 \mathrm{Dec}-$ laration of Helsinki and its later amendments or comparable ethical standards.

Informed consent Informed consent was obtained from all individual participants included in the study.

Human and animal rights This article does not contain any studies involving animals performed by any of the authors.
Retrospective studies Formal consent is not required for this type of study.

Open Access This article is licensed under a Creative Commons Attribution 4.0 International License, which permits use, sharing, adaptation, distribution and reproduction in any medium or format, as long as you give appropriate credit to the original author(s) and the source, provide a link to the Creative Commons licence, and indicate if changes were made. The images or other third party material in this article are included in the article's Creative Commons licence, unless indicated otherwise in a credit line to the material. If material is not included in the article's Creative Commons licence and your intended use is not permitted by statutory regulation or exceeds the permitted use, you will need to obtain permission directly from the copyright holder. To view a copy of this licence, visit http://creativecommons.org/licenses/by/4.0/.

\section{References}

1. Bushnell BD, Bynum DK (2007) Malunion of the distal radius. J Am Acad Orthop Surg 15:27-40. https://doi.org/10.5435/00124 635-200701000-00004

2. Fernandez DL (1982) Correction of post-traumatic wrist deformity in adults by osteotomy, bone-grafting, and internal fixation. $\mathrm{J}$ Bone Joint Surg Am 64:1164-1178

3. Vroemen JC, Dobbe JGG, Jonges R, Strackee SD, Streekstra GJ (2012) Three-dimensional assessment of bilateral symmetry of the radius and ulna for planning corrective surgeries. J Hand Surg 37A:982-988. https://doi.org/10.1016/j.jhsa.2011.12.035

4. Ma B, Kunz M, Gammon B, Ellis RE, Pichora DR (2014) A laboratory comparison of computer navigation and individualized guides for distal radius osteotomy. Int J Comput Assist Radiol Surg 9:713-724. https://doi.org/10.1007/s11548-013-0966-8

5. Buijze GA, Prommersberger KJ, González Del Pino J, Fernandez DL, Jupiter JB (2012) Corrective osteotomy for combined intra- and extra-articular distal radius malunion. J Hand Surg Am 37:2041-2049. https://doi.org/10.1016/j.jhsa.2012.07.013

6. Honigmann P, Thieringer F, Steiger R, Haefeli M, Schumacher R, Henning J (2016) A Simple 3-dimensional printed aid for a corrective palmar opening wedge osteotomy of the distal radius. J Hand Surg Am 41:464-469. https://doi.org/10.1016/j.jhsa.2015.12.022

7. Schweizer A, Fürnstahl P, Nagy L (2013) Three-dimensional correction of distal radius intra-articular malunions using patientspecific drill guides. J Hand Surg Am 38:2339-2347. https://doi. org/10.1016/j.jhsa.2013.09.023

8. Banks J (2013) Adding value in additive manufacturing: researchers in the United Kingdom and Europe look to 3D printing for customization. IEEE Pulse 4:22-26. https://doi.org/10.1109/ MPUL.2013.2279617

9. Caiti G, Dobbe JGG, Strijkers GJ, Strackee SD, Streekstra GJ (2018) Positioning error of custom 3D-printed surgical guides for the radius: influence of fitting location and guide design. Int J Comput Assist Radiol Surg 13:507-518. https://doi.org/10.1007/ s11548-017-1682-6

10. Tack P, Victor J, Gemmel P, Annemans L (2016) 3D-printing techniques in a medical setting: a systematic literature review. Biomed Eng Online 15:115. https://doi.org/10.1186/s12938-016-0236-4

11. Dobbe JGG, Caiti G, Schreurs AW, Strackee SD, Beerens M, Streekstra GJ (2018) Positioning accuracy of a patient-tailored rimmed wedge implant for corrective osteotomy of the distal radius. Med Eng Phys 58:41-46. https://doi.org/10.1016/j.meden gphy.2018.05.001 
12. Wei X-M, Sun Z-Z, Rui Y-J, Song X-J, Jiang W-M (2016) Minimally invasive percutaneous plate osteosynthesis for distal radius fractures with long-segment metadiaphyseal comminution. Orthop Traumatol Surg Res 102:333-338. https://doi.org/10.1016/J. OTSR.2015.12.024

13. Taleb C, Zemirline A, Lebailly F, Bodin F, Facca S, Gouzou S, Liverneaux P (2015) Minimally invasive osteotomy for distal radius malunion: a preliminary series of 9 cases. Orthop Traumatol Surg Res 101:861-865. https://doi.org/10.1016/j. otsr.2015.07.016

14. Oh JK, Hwang JH, Sahu D, Jun SH (2011) Complication rate and pitfalls of temporary bridging external fixator in periarticular comminuted fractures. Clin Orth Surg 3:62-68. https://doi. org/10.4055/cios.2011.3.1.62

15. Smith EJ, Allan G, Gammon B, Sellens RW, Ellis RE, Pichora DR (2014) Investigating the performance of a wrist stabilization device for image-guided percutaneous scaphoid fixation. Int J Comput Assist Radiol Surg 9:155-164. https://doi.org/10.1007/ s11548-013-0834-6

16. Liverneaux PA, Gherissi A, Stefanelli MB (2008) Kirschner wire placement in scaphoid bones using fluoroscopic navigation: a cadaver study comparing conventional techniques with navigation. Int J Med Robot Comput Assist Surg 4:165-173. https://doi. org/10.1002/rcs. 194

17. Niederwanger C, Widmann G, Knoflach M, Schullian P, Hoermann R, Bale R (2013) Kirschner wire placement in scaphoid bones using intraoperative CT-guided stereotaxy. Minim Invasive Ther Allied Technol 22:165-170. https://doi.org/10.3109/13645 706.2012.724688

18. Walenkamp MMJ, de Muinck Keizer RJO, Dobbe JGG, Streekstra GJ, Goslings JC, Kloen P, Strackee SD, Schep NWL (2015) Computer-assisted 3D planned corrective osteotomies in eight malunited radius fractures. Strateg Trauma Limb Reconstr 10:109-116. https://doi.org/10.1007/s11751-015-0234-2

19. Dobbe JGG, Strackee SD, Schreurs AW, Jonges R, Carelsen B, Vroemen JC, Grimbergen CA, Streekstra GJ (2011) Computer-assisted planning and navigation for corrective distal radius osteotomy, based on pre- and intraoperative imaging. IEEE Trans Biomed Eng 58:182-190. https://doi.org/10.1109/ TBME.2010.2084576

20. Dobbe JGG, Vroemen JC, Strackee SD, Streekstra GJ (2013) Corrective distal radius osteotomy: including bilateral differences in 3-D planning. Med Biol Eng Comput 51:791-797. https://doi. org/10.1007/s11517-013-1049-2

21. Dobbe JGG, Vroemen JC, Strackee SD, Streekstra GJ (2013) Patient-tailored plate for bone fixation and accurate 3D positioning in corrective osteotomy. Med Biol Eng Comput 51:19-27. https:// doi.org/10.1007/s11517-012-0959-8

22. Caiti G, Dobbe JGG, Loenen ACY, Beerens M, Strackee SD, Strijkers GJ, Streekstra GJ (2018) Implementation of a semiautomatic method to design patient-specific instruments for corrective osteotomy of the radius. Int J Comput Assist Radiol Surg. https://doi. org/10.1007/s11548-018-1896-2

23. Dobbe JGG, de Roo MGA, Visschers JC, Strackee SD, Streekstra GJ (2018) Evaluation of a quantitative method for carpal motion analysis using clinical 3D and 4D CT protocols. IEEE Trans Med Imag. https://doi.org/10.1109/TMI.2018.2877503

24. Polytechnic University 3D Printing Case Study I Stratasys. https ://www.stratasys.com/resources/search/case-studies/polytechni c-university. Accessed 17 Aug 2019

25. Kuo HY, Su HR, Lai SH, Wu CC (2014) 3D object detection and pose estimation from depth image for robotic bin picking. IEEE Int Conf Autom Sci Eng 2014-Janua: 1264-1269. https:// doi.org/10.1109/CoASE.2014.6899489

26. Benítez Brito N, Suárez Llanos JP, Fuentes Ferrer M, Oliva García JG, Delgado Brito I, Pereyra-García Castro F, Caracena Castellanos N, Acevedo Rodríguez CX, Palacio Abizanda E (2016) Relationship between mid-upper arm circumference and body mass index in inpatients. PLoS ONE 11:e0160480. https://doi. org/10.1371/journal.pone. 0160480

27. Dobbe JGG, Kievit AJ, Schafroth MU, Blankevoort L, Streekstra GJ (2014) Evaluation of a CT-based technique to measure the transfer accuracy of a virtually planned osteotomy. Med Eng Phys 36:1081-1087. https://doi.org/10.1016/j.medengphy.2014.05.012

28. The Practical Guide Identification, Evaluation, and Treatment of Overweight and Obesity in Adults NHLBI Obesity Education Initiative

29. Li J, Lin J, Yang Y, Xu J, Fei Q (2018) 3-Dimensional printing guide template assisted percutaneous vertebroplasty: technical note. J Clin Neurosci 52:159-164. https://doi.org/10.1016/J. JOCN.2018.03.012

30. Yin HW, Xu J, Xu WD (2017) 3-Dimensional printing-assisted percutaneous fixation for acute scaphoid fracture: 1-shot procedure. J Hand Surg Am 42:301.e1-301.e5. https://doi.org/10.1016/j. jhsa.2017.01.017

Publisher's Note Springer Nature remains neutral with regard to jurisdictional claims in published maps and institutional affiliations. 